\title{
Pushing the Limits of Absolute Scale Energy Dispersive X-ray Quantification
}

Katherine E. MacArthur ${ }^{1 *}$, Sean M. Collins ${ }^{2}$, Hamish G. Brown ${ }^{3,4}$, Scott D. Findlay ${ }^{4}$, Leslie J. Allen ${ }^{5}$

1. The Ernst Ruska Centre for Microscopy and Spectroscopy with Electrons and the Peter Grünberg Institute Forschungszentrum Jülich, Jülich, Germany.

2. Department of Materials Science and Metallurgy, University of Cambridge, Cambridge, United Kingdom.

3. National Centre for Electron Microscopy, Molecular Foundry, Lawrence Berkeley National Laboratory, Berkeley, California

4. School of Physics and Astronomy, Monash University, Clayton, Australia.

5. School of Physics, University of Melbourne, Parkville, Australia.

* Corresponding author: k.macarthur@fz-juelich.de

The current state of the art in energy dispersive x-ray (EDX) spectroscopy combines larger solid angle silicon drift detectors (SDDs) with high brightness sources and sometimes aberration corrected electron beams. Such is the improvement in microscope resolution and the number of counts in the collected Xray signal that atomic resolution elemental maps [1] and EDX tomography [2] are both now possible on sufficiently dose resilient samples. Such data sets provide invaluable and unprecedented qualitative compositional information about materials which necessarily leads us to the question of how much can we quantitatively say about sample compositions at the nanometer and sub-nanometer scales?

A relatively new method [3] for EDX quantification, has been derived making use of the improved stability and capability of modern electron microscopes based on partial ionization cross sections. Based on a modification of the zeta-factor method, first proposed by Watanabe et al. [4], the method assumes linearity, i.e. the number of X-rays produced is linearly proportional to the number of atoms of a given element within the sample. This assumption holds where absorption, fluorescence and electron channelling are all negligible. The partial ionization cross section can be defined as the raw intensity, $I_{A}$, divided by the electron dose, $D$, the atomic density, $n_{A}$, sample thickness, $t$, and illumination area, $A$.

$$
\sigma_{A}=\frac{I_{A}}{D n_{A} t A}
$$

Such experimentally determined cross sections derived from single element standards of known geometry are microscope dependent, incorporating the effects of detector efficiency and collection angles and shadowing from the particular sample holder. One primary advantage of this quantification approach is that it yields the number of atoms of a particular element in a given illumination area, thereby yielding information about sample density. For catalyst nanoparticles it simultaneously provides particle size (in number of atoms) and composition from one experimental map [5].

Here, by advancing this EDX quantification method to incorporate stochiometric standards of arbitrary morphology with compressed sensing tomography algorithms, accurate compositional quantification is of glass blends is demonstrated on the nanometer scale. Such methodical developments are particularly beneficial for observing and accurately evaluating the degree of miscibility at interfaces.

Finally, the potential challenges and pitfalls of examining atomic resolution data will also be presented [6]. 
References:

[1] LJ Allen et al. MRS Bull. 32, (2012) p. 47-52.

[2] A Genc et al. Ultramic. 131, (2013) p. 24-32.

[3] KE MacArthur et al. Micosc. Microanal. 22, 01 (2016), p. 71-81.

[4] M Watanabe et al. Ultramic. 65, (1996) p. 187-198.

[5] KE MacArthur et al. Mater. Sci. Technol. 32, 3 (2016) p. 248-253.

[6] The authors acknowledge financial support from the German Helmholtz Foundation, the Henslow Research Fellowship and Girton College, Cambridge.
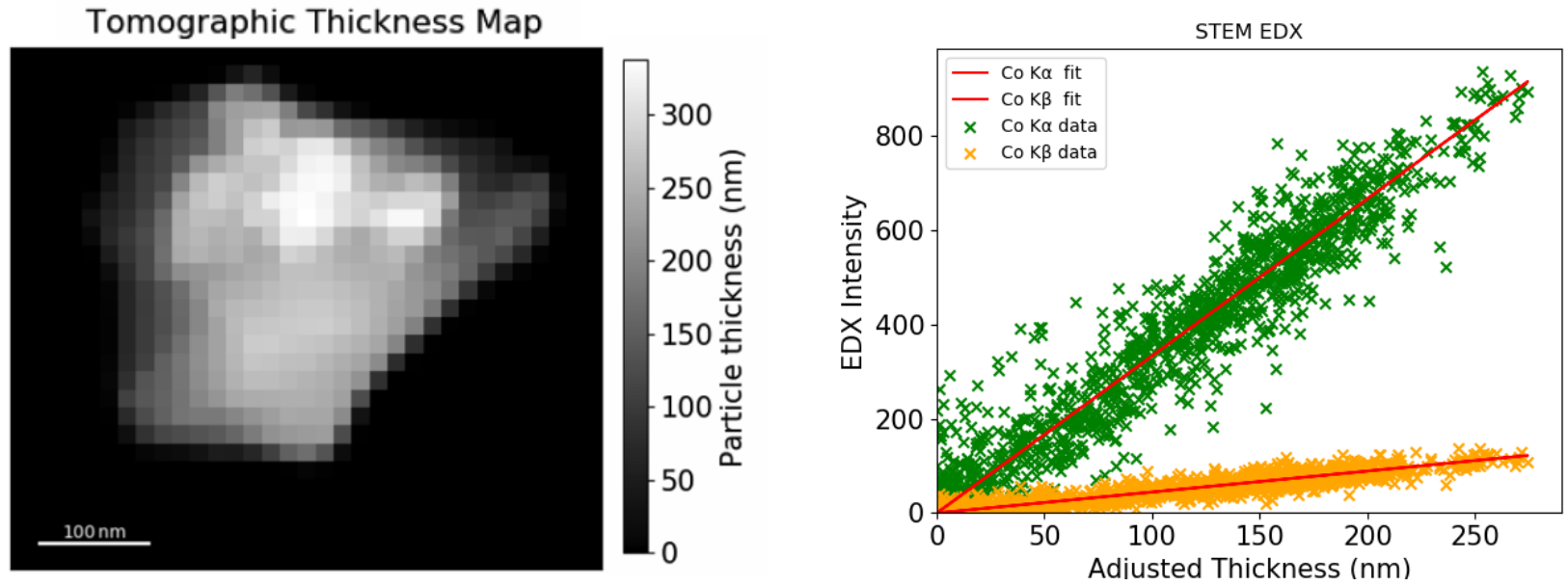

Figure 1. Example calibration data for X-ray partial ionization cross section determination. The thickness map of a nanoparticle with arbitrary shape determined from an ADF tomographic reconstruction (left). The resultant intensity-thickness plot for cross section determination (right). 